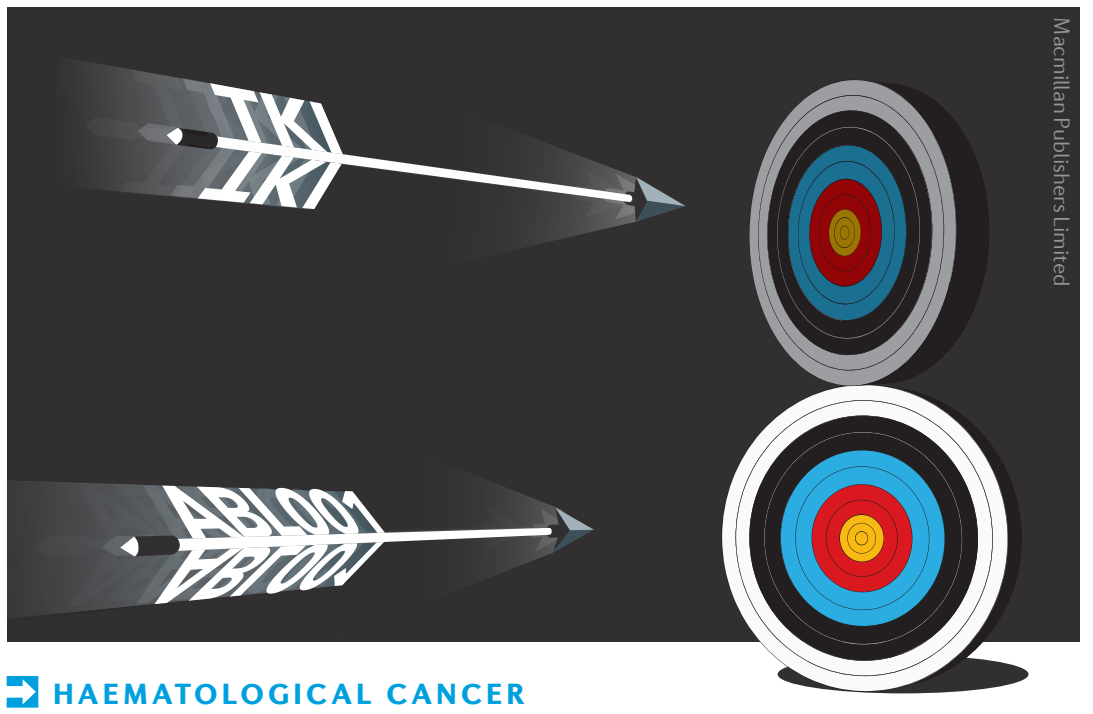

\title{
Dual targeting to defeat resistance
}

Following the advent of tyrosine-kinase inhibitors (TKIs) targeting the BCR-ABL1 fusion protein, the prognosis of patients with chronic myeloid leukaemia $(\mathrm{CML})$ has improved. The TKIs currently administered to these patients block the catalytic activity of BCR-ABL1 through competition with ATP. ABL001, an inhibitor with a different mechanism of action, has been developed by Andrew Wylie and colleagues, and results of preclinical studies with this agent have now been published.

"Patients with CML require daily medication for life. Some patients experience adverse effects, and others develop resistance to therapy through mutations in the binding site," Wylie summarizes. Thus, ABL001 was designed as an allosteric inhibitor to force BCR-ABL1 into an inactive conformation upon binding. This approach, Wylie explains, "not only provides a more-comprehensive coverage of the target, but should also prevent resistance because the appearance of mutations in one binding site would be insufficient to prevent inhibition by the other combination partner."

The investigators carried out experiments with KCL-22 (CML-derived) mouse xenografts. In these mice, treatment with either ABL001 or the TKI nilotinib led to rapid tumour regression but, eventually, clones harbouring mutations in residues critical for enzyme inhibition emerged. In mice with resistant tumours, a treatment switch to the other agent led to a second response, which was only transient. By contrast, mice initially treated

mice treated with...

combination

had complete

tumour regressions

without

recurrence, even after

cessation with ABL001 and nilotinib in combination had complete tumour regressions without recurrence, even after treatment cessation.

Wylie and colleagues are currently testing ABL001 in a phase I trial as a single-agent, as well as in combination with several TKIs, in patients with prior resistance to, or intolerance of, treatment with at least two other TKIs. The aim, reveals Wylie is "to reach the day when patients with CML can safely stop taking pills without experiencing disease progression."

Diana Romero

ORIGINAL ARTICLE Wylie, A. A. et al. The allosteric inhibitor ABL001 enables dual targeting of BCR-ABL1. Nature http://dx.doi.org/10.1038/nature21702 (2017) FURTHER READING Rosti, G., Castagnetti, F., Gugliotta, G. and Baccarani, M. Tyrosine kinase inhibitors in chronic myeloid leukaemia: which, when, for whom? Nat. Rev. Clin. Oncol. 14, 141-154 (2017) 